\title{
A DIRECT PROOF OF LEUTBECHER'S LEMMA
}

\author{
by KLAUS WOHLFAHRT \\ (Received 23 December, 1970)
}

Using the theory of group extensions, A. Leutbecher [1] proved this

Lemma. Let $G$ be a group, and w some 2-cocycle of a trivial G-module $M$. The cohomology class of $w$ will contain symmetric cocycles if and only if $w$ is semisymmetric.

Here we have called $w$ symmetric or semisymmetric according as $w(h, g)=w(g, h)$ for all $g, h \in G$ or only for those with $h g=g h$. In one direction, the proof reduces to observing that 2-coboundaries of trivial $G$-modules are semisymmetric. The nontrivial part of the lemma also admits of a straightforward proof, as follows.

If $w$ is any cocycle of a trivial $G$-module, following H. Petersson [2, p. 47] we define, inductively for $n \geqq 2$,

$$
w\left(g_{1}, \ldots, g_{n}, g_{n+1}\right)=w\left(g_{1}, \ldots, g_{n}\right)+w\left(g_{1} g_{2} \ldots g_{n}, g_{n+1}\right)
$$

for $g_{j} \in G(1 \leqq j \leqq n+1)$. Then

$$
w\left(g_{1}, \ldots, g_{j}, g_{j+1}, \ldots, g_{n}\right)=w\left(g_{1}, \ldots, g_{j} g_{j+1}, \ldots, g_{n}\right)+w\left(g_{j}, g_{j+1}\right) .
$$

If, in particular, $g_{j+1}=h^{-1}$ is inverse to $g_{j}=h$, and if $e$ denotes the neutral element of $G$, we have

$$
\begin{aligned}
w\left(g_{1}, \ldots, h, h^{-1}, \ldots, g_{n}\right) & =w\left(g_{1}, \ldots, e, \ldots, g_{n}\right)+w\left(h, h^{-1}\right) \\
& =w\left(g_{1}, \ldots, g_{j-1}, g_{j+2}, \ldots, g_{n}\right)+w(e, e)+w\left(h, h^{-1}\right),
\end{aligned}
$$

where we have used $w(g, e)=w(e, g)=w(e, e)(g \in G)$. then

Proposition 1. If $w$ is semisymmetric and if $f, g, h \in G$ are such that $f$ commutes with $h^{-1} g$,

$$
w\left(h, f, h^{-1}\right)-w\left(h, h^{-1}\right)=w\left(g, f, g^{-1}\right)-w\left(g, g^{-1}\right) .
$$

Indeed, semisymmetry gives $w\left(h^{-1}, g, f\right)=w\left(f, h^{-1}, g\right)$, and so

$$
\begin{aligned}
w\left(h, f, h^{-1}\right)-w\left(h, h^{-1}\right) & =w\left(h, h^{-1} g f g^{-1} h, h^{-1}\right)-w\left(h, h^{-1}\right) \\
& =w\left(h, h^{-1}, g, f, g^{-1}, h, h^{-1}\right)-w\left(h^{-1}, g, f, g^{-1}, h\right)-w\left(h, h^{-1}\right) \\
& =w\left(h, h^{-1}\right)+2 w(e, e)+w\left(g, f, g^{-1}\right)-w\left(f, h^{-1}, g, g^{-1}, h\right) \\
& =w\left(g, f, g^{-1}\right)-w\left(g, g^{-1}\right),
\end{aligned}
$$

where we have used $w\left(h^{-1}, h\right)=w\left(h, h^{-1}\right)(h \in G)$. Proposition 1 is thus established.

Proposition 2. If $w$ is semisymmetric, then there is a 1-cochain $c: G \rightarrow M$ such that

$$
c(h g)-c(g h)=w(h, g)-w(g, h) \quad(g, h \in G) .
$$

To prove this we choose, using Zermelo's axiom, a representative in each class of 
conjugate elements of $G$ and then define, consistently according to Proposition $1, c$ on the class of $f \in G$ by

$$
c\left(g f g^{-1}\right)=w\left(g, f, g^{-1}\right)-w\left(g, g^{-1}\right) \quad(g \in G) .
$$

If $g, h \in G$ are such that $g h$ is in the class represented by $f$, we have

$$
g h=u f u^{-1}, \quad h g=h u f u^{-1} h^{-1}
$$

for some $u \in G$. Then

$$
\begin{aligned}
c(h g)-c(g h) & =w\left(h u, f, u^{-1} h^{-1}\right)-w\left(h u, u^{-1} h^{-1}\right)-w\left(u, f, u^{-1}\right)+w\left(u, u^{-1}\right) \\
& =w\left(h, u, f, u^{-1}, h^{-1}\right)-w\left(h, u, u^{-1}, h^{-1}\right)-w\left(u, f, u^{-1}\right)+w\left(u, u^{-1}\right) \\
& =w\left(h, u f u^{-1}, h^{-1}\right)-w\left(h, h^{-1}\right)-w(e, e) \\
& =w\left(h, g, h, h^{-1}\right)-w(g, h)-w\left(h, h^{-1}\right)-w(e, e) \\
& =w(h, g)-w(g, h)
\end{aligned}
$$

so Proposition 2 is proved.

To establish the lemma, we derive from $c$ the 2-coboundary $b=\partial c$,

$$
b(g, h)=c(g)+c(h)-c(g h) \quad(g, h \in G) .
$$

Then, by Proposition 2,

$$
b(g, h)-b(h, g)=w(h, g)-w(g, h) \quad(g, h \in G),
$$

and $w+b$, which is cohomologous to $w$, will be symmetric.

\section{REFERENCES}

1. A. Leutbecher, Uber Automorphiefaktoren und die Dedekindschen Summen, Glasgow Math. J. 11 (1970), 41-57. $23-67$.

2. H. Petersson, Zur analytischen Theorie der Grenzkreisgruppen, Teil I, Math. Ann. 115 (1937),

MATHEMATISCHES INSTITUT

HEIDELBERG

GERMANY 